Maciej Urlik', Konstanty Szułdrzyński' ${ }^{2,3}$, Tomasz Stącel', Mirosław Nęcki', Piotr Bielański',

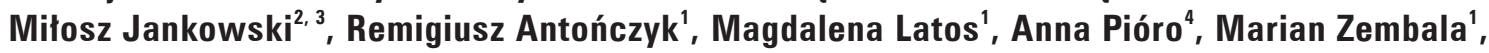
Krzysztof Pyrć ${ }^{5}$, Marek Ochman ${ }^{1}$

'Department of Cardiac, Vascular and Endovascular Surgery and Transplantology, Silesian Center for Heart Diseases in Zabrze,

Medical University of Silesia, Katowice, Poland

2Jagiellonian University Medical College, Faculty of Medicine, Krakow, Poland

${ }^{3}$ The Centre for Extracorporeal Therapies University Hospital Krakow, Krakow, Poland

${ }^{4}$ Department of Cardiac Anaesthesia and Intensive Care SUM, Silesian Centre for Heart Diseases, Zabrze, Poland

${ }^{5}$ Virogenetics Laboratory of Virology, Malopolska Centre of Biotechnology, Jagiellonian University, Krakow, Poland

\title{
First lung transplantation as a treatment of a patient supported with extracorporeal membrane oxygenation (ECMO) after COVID-19 in Poland
}

\begin{abstract}
A 44-year-old male with no history of underlying diseases was referred to academic hospital due to ARDS with confirmed SARSCoV-2 infection after 7 days of mechanical ventilation. Veno-venous (VV) extracorporeal membrane oxygenation (ECMO) was initiated as no improvement was noted in prone position. Mechanical ventilation was continued with TV of 3-4 mL/ $/ \mathrm{kg}$. A gradual decline of static lung compliance was observed from baseline $35 \mathrm{~mL} / \mathrm{cm} \mathrm{H}_{2} 0$ to $8 \mathrm{~mL} / \mathrm{cm} \mathrm{H}_{2} \mathrm{O}$. The chest CT scan revealed extensive ground-glass areas with a significant amount of traction bronchiectasis after 3 weeks since admission. When the patient was negative for SARS-CoV-2 during the $4^{\text {th }}$ week of ECM0, the decision to perform an emergency lung transplantation (LTX) was made based on the ongoing degradation of lung function and irreversible damage to lung structure. The patient was transferred to the transplant center where he was extubated, awaiting the transplant on passive oxygen therapy and ECMO. Double lung transplantation was performed on the day $30^{\text {th }}$ of ECMO. Currently, the patient is self-reliant. He does not need oxygen therapy and continues physiotherapy.

ECMO may be life-saving in severe cases of COVID-19 ARDS but some of these patients may require LTx, especially when weaning proves impossible. VV ECMO as a bridging method is more difficult but ultimately more beneficial due to insufficient number of donors, and consequently long waiting time in Poland.
\end{abstract}

Key words: lung transplantation, COVID-19, SARS-CoV-2, extracorporeal membrane oxygenation

Adv Respir Med. 2021; 89: 328-333

\section{Introduction}

The severe acute respiratory syndrome coronavirus 2 (SARS-CoV-2) causes respiratory infection of variable severity known as coronavirus disease (COVID-19). Some of the patients with acute respiratory failure may require extracorporeal membrane oxygenation (ECMO) [1]. ECMO may not only be a salvage therapy in profound hypoxemia and an efficient tool to provide lung-protective ventilation but may also serve as an effective bridge to lung transplantation (LTx) [2].

\section{Case presentation}

A 44-year-old male with no history of underlying chronic illness was admitted to the intensive care ECMO unit due to acute respiratory failure caused by COVID-19 infection. Detailed course of the disease is presented in Figure 1. He was treated with remdesivir, convalescent plasma 


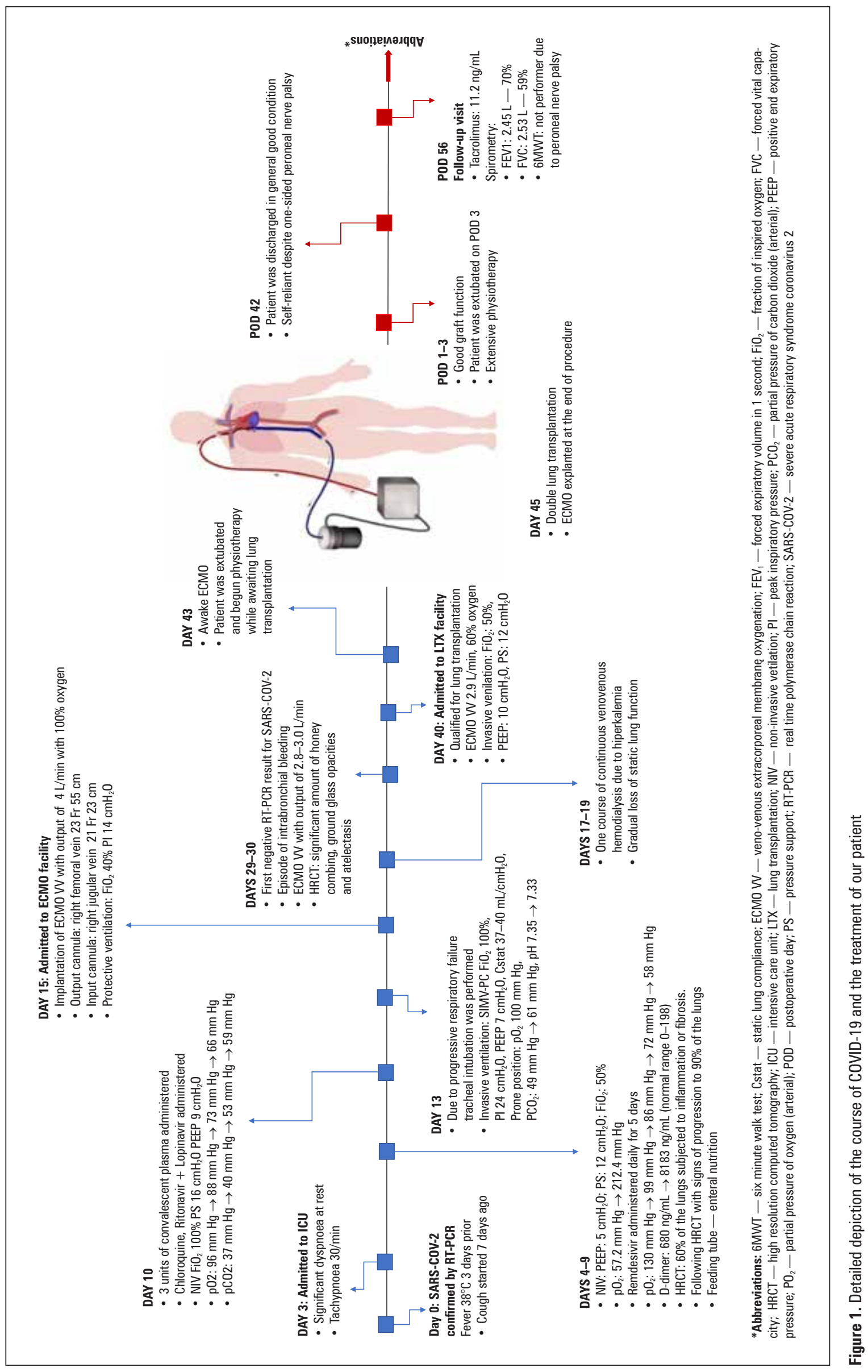

www.journals.viamedica.pl 

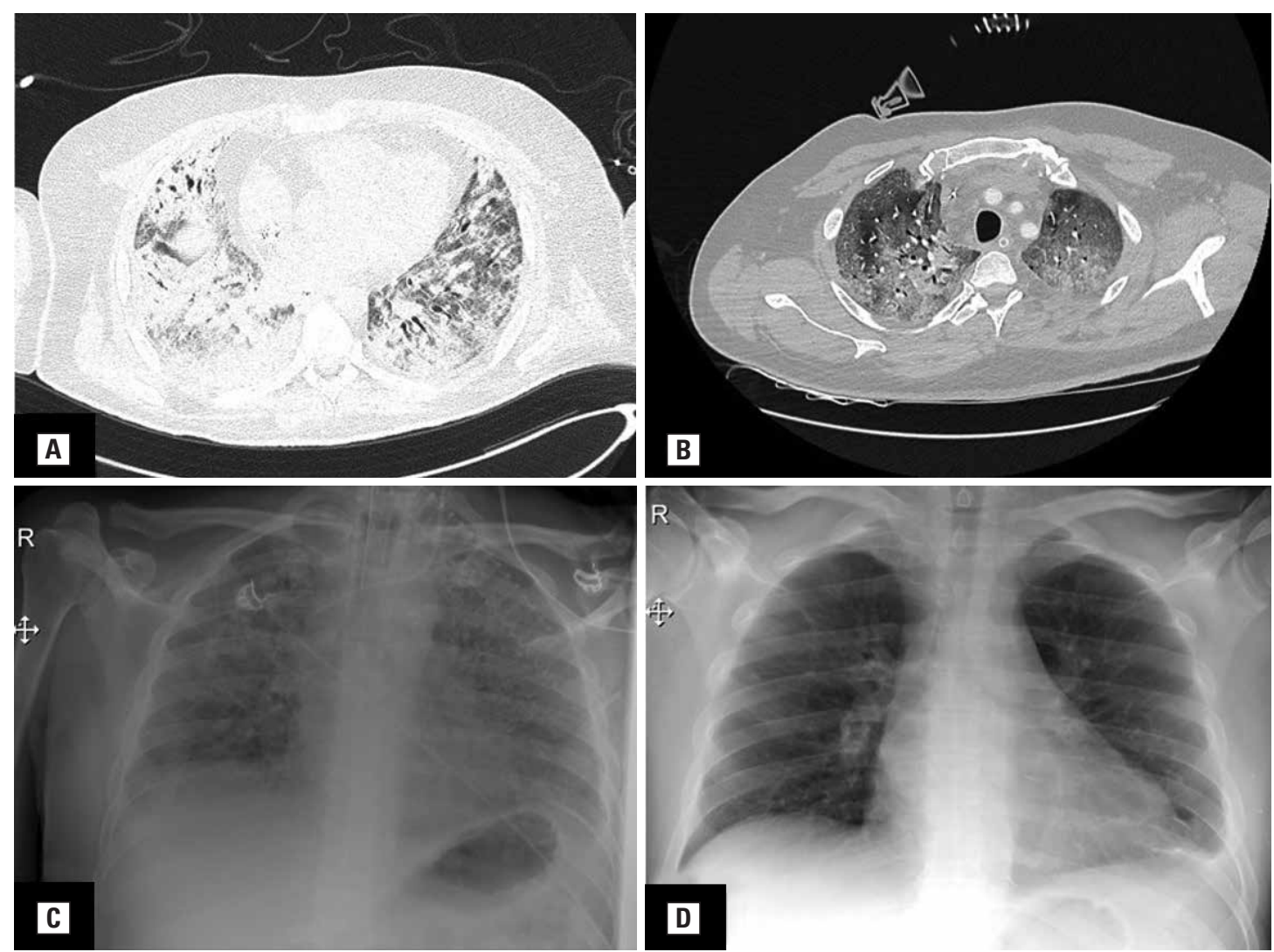

Figure 2. A, B. High-resolution computed tomography of the thorax presenting significant amount of lungs subjected to inflammation and fibrosis; C. Chest X-ray of the patient treated with invasive ventilation alone due to COVID-19; D. Chest X-ray at discharge from the lung transplant facility

and ritonavir + lopinavir with no improvement. He required invasive mechanical ventilation for 7 days with the tidal volume of $6 \mathrm{~mL} / \mathrm{kg}$, positive end-expiratory pressure (PEEP) $14 \mathrm{~cm} \mathrm{H}_{2} \mathrm{O}$, frequency of $22 / \mathrm{min}$ and fraction of inspired oxygen $\left(\mathrm{FiO}_{2}\right)$ 1.0. After placing him in prone position, minor improvement of oxygenation was noted with a marked increase of arterial partial pressure of carbon dioxide $\left(\mathrm{PaCO}_{2}\right)$ and a respiratory acidosis. Veno-venous (VV) ECMO with femoro-jugular access was instituted due topersistent respiratory acidosis, oxygenation index of $80 \mathrm{~mm} \mathrm{Hg}$ and discouraging response to proning. Mechanical ventilation was switched to protective settings. His hemodynamic status improved significantly. Acute kidney injury resolved after a single course of continuous renal-replacement therapy. A gradual loss of static lung compliance was observed from $35 \mathrm{~mL} / \mathrm{cm} \mathrm{H}_{2} \mathrm{O}$ on admission to the final $8 \mathrm{~mL} / \mathrm{cm} \mathrm{H}_{2} \mathrm{O}$. Transient hemorrhage to the left main bronchus ceased after tapering heparin infusion to the activated partial thromboplastin time (APTT) level of around 40 sec. (baseline 22 sec.). High-resolution computed tomography (HRCT) scan of the thorax revealed extensive ground-glass opacities with a significant amount of traction bronchiectasis. A detailed depiction is presented in Figure 2. The patient turned negative for SARS-CoV-2 after 3 weeks of treatment. He was awake, alert and cooperative after cessation of sedation. The decision to perform an emergency lung transplantation was made during the fourth week on ECMO due to irreversible lung failure. The man was subsequently transferred to the transplant center (blood flow $3.0 \mathrm{~L} / \mathrm{min}$ ) while on mechanical ventilation and ECMO. He was extubated in the following days according to our awake ECMO protocol. Sequential double lung transplantation was performed on day $30^{\text {th }}$ since the initiation of ECMO. The procedure itself took place while the patient was still treated with ECMO support. He was hemodynamically unstable with periodical support of catecholamines but with left ventricle ejection fraction (LVEF) at $65 \%$ without pulmonary hypertension. Pulmonary artery catheter Swan-Ganz (PAC), which is standard monitoring in LTx, was not placed in the patient because of inadequate measurement during VV-ECMO. A transesophageal echocardiography (TEE) probe was placed for TEE monitoring. Anesthesia and analgesia were approached in a manner typical 
of the lung transplantation procedure. The patient was intubated with a left-sided Robertshaw double-lumen tube (DLT). The most critical steps during lung transplantation was induction of anesthesia, pulmonary artery clamping, graft implantation and reperfusion. Initial ventilator settings were set according to lung protective ventilation strategy. VV-ECMO was continued during the whole procedure. Inhaled nitric oxide (iNO) was administered - 60 ppm. Pleural cavities were accessed via mini-thoracotomy through the $5^{\text {th }}$ intercostal space, but higher access seems to be more reasonable as those patients have their diaphragm located higher. Typical preparation of the lungs and hila was carried out. The visceral pleura was very fragile as well as easy to bleed even at careful preparation due to dense vascular net. Morphology of the lungs presented atypical findings of cysts filled with dense hemolyzed blood as depicted in Figure 3. Histopathological findings of the lungs were presented in Figure 4. ECMO was explanted after the implantation of both lungs. The authors of this work advise to pay special attention to the fact that respiratory infection in the course of COVID-19 may shrink the volume of the chest cavity. Proper sizing of 1:1 or less is crucial during qualification. During the procedure, there was a need for surgical lung volume reduction using linear automatic staplers. The authors took the liberty of more liberal size-matching of the lungs due to critical condition of the patient and increasing risk of ECMO-related complications. The man was admitted to the lung transplantation ward intubated, in stable condition with pressure controlled synchronized intermittent mechanical ventilation (PC-SIMV) with PEEP of $8 \mathrm{~mm} \mathrm{H}_{2} \mathrm{O}$, frequency of $12 / \mathrm{min}$ and peak pressure $24 \mathrm{~mm} \mathrm{H}_{2} \mathrm{O}$.

Immunosuppression was initiated at the beginning of the operation with venous infusion of tacrolimus as well as $1 \mathrm{~g}$ of methylprednisolone followed by $125 \mathrm{mg}$ every 12 hours 3 times. Standard maintenance immunosuppression consisted of tacrolimus (target concentration of 10-12 ng/mL), mycofenolate mofetil twice a day and prednisolone. The rest of the drugs were administered according to standard protocol. The patient was extubated on the third postoperative day (POD). Extensive physical rehabilitation was implemented since the first POD. First two weeks were uneventful except for partial impairment of the right common fibular nerve. The patient obtained $65 \%$ of predicted forced expiratory volume in 1 second 4 weeks after the double lung transplantation. Proper healing was observed

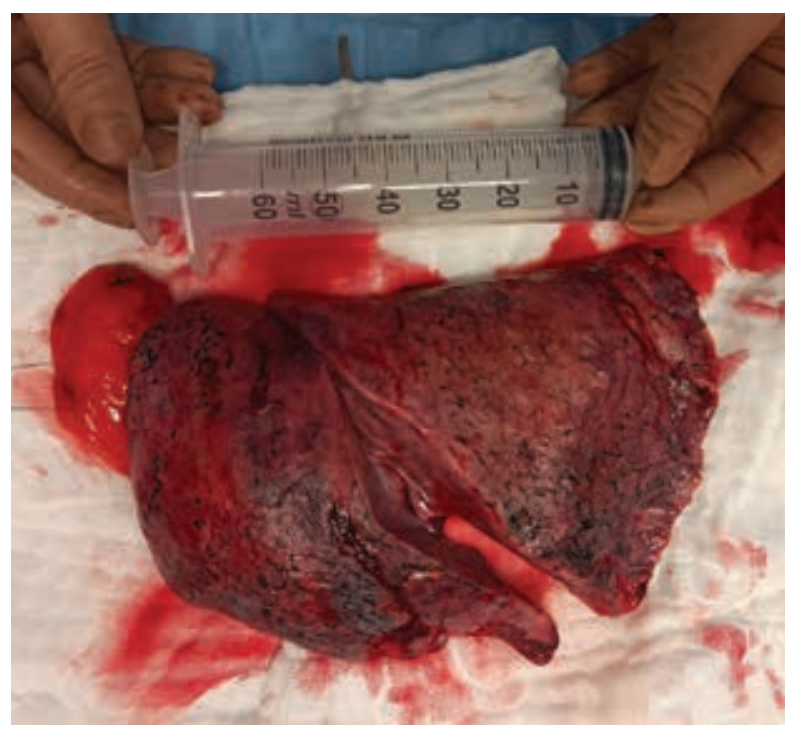

Figure 3. A photograph of the recipient's native lung minutes after the explantation

under bronchoscopic evaluations. He was discharged home.

We report this case now knowing that the patient has already reached a 3-month follow-up in general good condition with full respiratory capacity, as we can assess the treatment to be successful when the patient achieves this stage.

\section{Discussion}

A significant proportion of patients with COVID-19 develop respiratory failure ranging from mild dyspnea treated with oxygen therapy to severe acute respiratory distress syndrome (ARDS) fulfilling criteria for ECMO support. Experience with extracorporeal gas exchange gathered in 2009 and 2010 together with the first randomized trial revealing benefit of ECMO in ARDS [3] led to its widespread use in the intensive care. However, the outcome of ECMO in SARS-CoV-2-associated ARDS has not been clearly defined yet. The death in COVID-19 may arise predominantly from the multiorgan failure amidst the cytokine storm [4] with a very little fraction of patients dying of profound hypoxemia [5]. Therefore, ECMO is indicated mainly in subjects with predominant respiratory failure, preferably without the involvement of other organs [1]. The ultimate result of ECMO is determined by the ability to provide more protective mechanical ventilation and appropriate gas exchange on the one hand and the risk of complications and the chance of good long-term lung function on the other. 

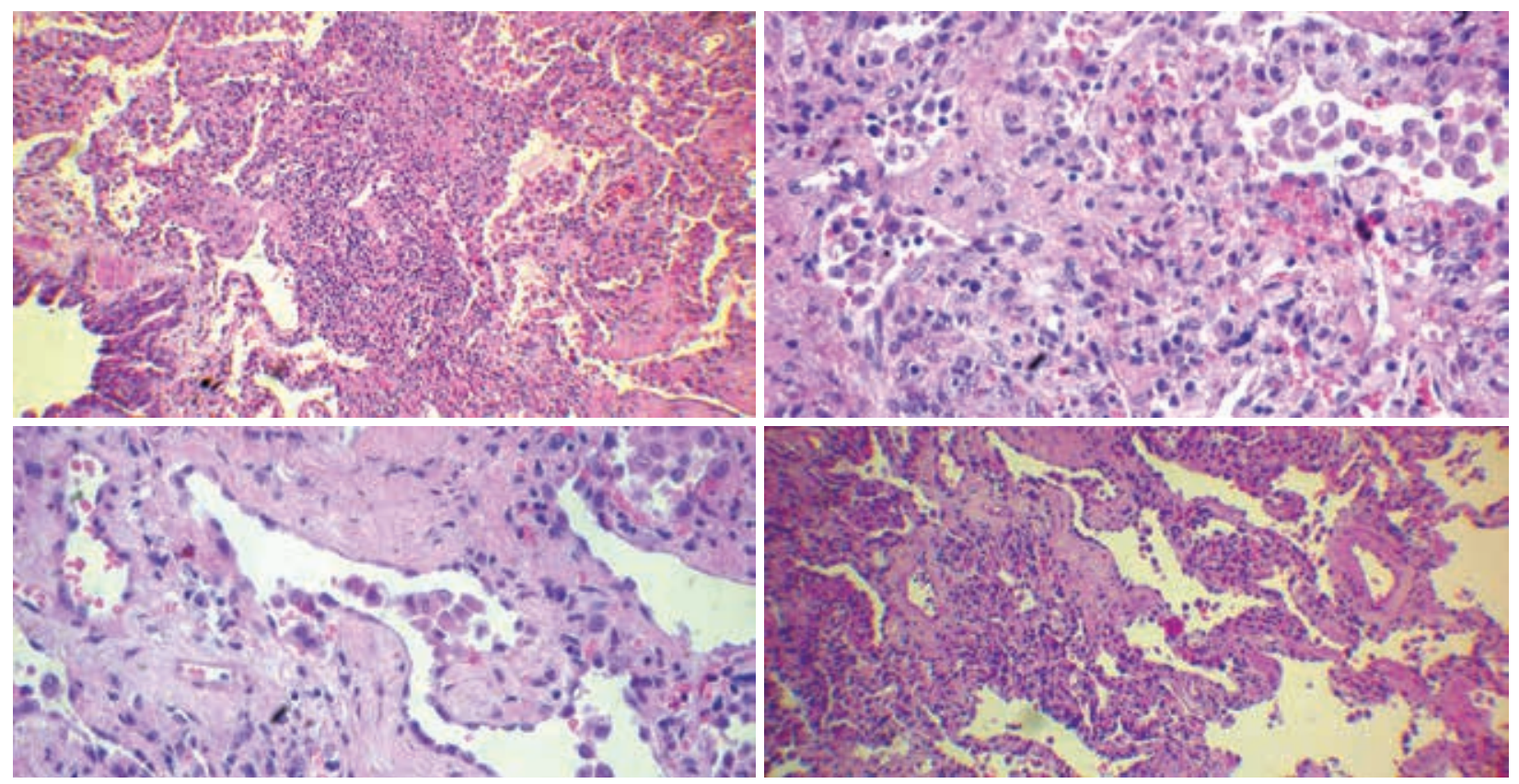

Figure 4. Histopathological examinations of native lungs: interstitial pneumonia with fibrosis; focal proliferation of type 2 pneumocytes; focal squamous metaplasia of bronchial epithelium; obliteration of some small arterioles; organized exudate in some of the alveoli

Intense lung fibrosis has been described in some COVID-19 patients ending up in a severely decreased functional capacity [6]. This case has shown that advanced fibrosis may develop over as little as 4-5 weeks despite lung-protective ventilation. It also demonstrates that patients may require emergency lung transplantation in order to be weaned off ECMO. It is also possible that prolonged period of non-invasive ventilation with increasing both respiratory effort and support levels may have led to progression of the patient's self-inflicted lung injury [7]. Simultaneously, proper assessment of neurological function and obtaining an informed consent for the transplantation warrants extubation which may be facilitated with ECMO. Therefore, this case is a proof that ECMO may be a valuable option as a bridge to transplant in selected patients with COVID-19 ARDS.

However, careful consideration must be applied when it comes to qualifying patients to be treated by means of lung transplantation [8]. The patient should present no other significant organ failure. Decision to perform lung transplantation among individuals qualified due to postCovid-19 respiratory failure should be made timely. It is in the patients' best interests to be able to survive without a transplant. Hence, infected lungs should be allowed to heal first, regardless of the extent of respiratory support including ECMO. The presented case is the first double lung transplantation due to COVID-19 among all lung transplantations performed in our facility as well as in Poland. The authors would like to underline that at the date of double lung transplantation (end of July 2020), there was only 5 similar cases described in the literature. There are reports of 5 lung transplantations performed shortly after obtaining the positive-turned negative virology in China [9], [10]even those whose nucleic acid test results had turned negative and those receiving maximal medical support, have been noted to progress to irreversible fatal respiratory failure. Lung transplantation (LT. Similarly to our patient, all of them required ECMO as a bridge to lung transplantation. In comparison to Chen et al., our patient had ECMO explanted in the surgical theatre after the procedure with a good outcome [9] even those whose nucleic acid test results had turned negative and those receiving maximal medical support, have been noted to progress to irreversible fatal respiratory failure. Lung transplantation (LT. Another interesting difference is that Chen's patients were operated via Clamshell approach, while our patient underwent the procedure through double minithoracotomy. This finding is important because it is more beneficial for the patient mostly in terms of upcoming extensive physiotherapy. Two subjects described by Han et al. also required ECMO as a bridge to lung transplantation. They were 
operated on with a good outcome [10] and had the ECMO therapy withdrawn on respectively the fifth and second POD.

The great advantage of our case report is that it depicts the young patient without comorbidities whose lungs were permanently damaged to the point of needing lung transplantation. Such a finding is supported by a paper published by Bharat et al. who described successful lung transplantation of the 43-year-old male patient with well-controlled type 2 diabetes mellitus despite his pre-transplant course being more complicated than in case of our patient. Bharat et al. also reported even younger case (a 28-year-old woman) [11]. However, it is worth mentioning that she was receiving immunosuppressive treatment due to her preexisting conditions. In our facility, it is vital that potential lung transplant candidate obtains negative SARS-CoV-2 real-time polymerase chain reaction (RT-PCR) test result. This condition was met before listing of our patient as well as the next patient transplanted by our team due to post-COVID-19 ARDS several weeks later. Such a thing was not necessary in Klinikum Klagenfurt am Wörthersee, where the first case of lung transplantation for a patient with a persistently positive SARS-CoV-2 RT-PCR test result took place [12]. A 44-year-old woman with mild psoriatic arthritis and idiopathic CD4 lymphocytopenia became a lung transplant recipient as it is reported by Lang et al. from the aforementioned Austrian facility. This case report is particularly important because it shows certain limitation of the RT-PCR testing. This method may produce a positive result based on nucleic acid segments of residual virus without actual infectivity as indicated by exceptionally high RT-PCR cycle threshold values. The Austrian team determined that there was no active infection based on negative Vero cell cultures result. The authors of this paper advice careful consideration and very thorough process of excluding active infection among patients with positive RT-PCR result for SARS-CoV-2. ECMO VV in case of our patient was safely discontinued at the end of the procedure. It shows that since the first POD such patients do not have to endure the risk of adverse effects associated with this therapy either. Poland among many countries is facing the insufficient number of lung donors, especially during the time of pandemics. Therefore, VV ECMO is more beneficial despite being the more challenging in terms of patient maintenance as a potential recipient has a greater chance of being successfully bridged to lung transplantation in comparison to VA ECMO patient.

\section{Acknowledgments}

The authors would like to thank Jerzy Nożyński MD for histopathological examination of the explanted lungs, as well as transplant coordinators involved with this procedure.

\section{Conflict of interest}

All authors report no conflict of interest regarding issues connected to this paper.

\section{References:}

1. Shekar K, Badulak J, Peek G, et al. Extracorporeal life support organization coronavirus disease 2019 interim guidelines: a consensus document from an International Group of Interdisciplinary Extracorporeal Membrane Oxygenation Providers. ASAIO J. 2020; 66(7): 707-721, doi: $10.1097 /$ MAT.0000000000001193, indexed in Pubmed: 32604322.

2. Gulack BC, Hirji SA, Hartwig MG. Bridge to lung transplantation and rescue post-transplant: the expanding role of extracorporeal membrane oxygenation. J Thorac Dis. 2014; 6(8): 1070-1079, doi: 10.3978/i.issn.2072-1439.2014.06.04, indexed in Pubmed: 25132974.

3. Peek G, Mugford M, Tiruvoipati R, et al. Efficacy and economic assessment of conventional ventilatory support versus extracorporeal membrane oxygenation for severe adult respiratory failure (CESAR): a multicentre randomised controlled trial. The Lancet. 2009; 374(9698): 1351-1363, doi: 10.1016/s01406736(09)61069-2.

4. Mehta P, McAuley D, Brown M, et al. COVID-19: consider cytokine storm syndromes and immunosuppression. The Lancet. 2020; 395(10229): 1033-1034, doi: 10.1016/s01406736(20)30628-0.

5. Grasselli G, Greco M, Zanella A, et al. Risk factors associated with mortality among patients with COVID-19 in intensive care units in Lombardy, Italy. JAMA Intern Med. 2020; 180(10): 1345-1355, doi: 10.1001/jamainternmed.2020.3539, indexed in Pubmed: $\underline{32667669}$.

6. George P, Wells A, Jenkins R. Pulmonary fibrosis and COVID-19 the potential role for antifibrotic therapy. The Lancet Respiratory Medicine. 2020; 8(8): 807-815, doi: 10.1016/s22132600(20)30225-3.

7. Marini J, Gattinoni L. Management of COVID-19 respiratory distress. JAMA. 2020; 323(22): 2329-2330, doi: 10.1186/cc2392.

8. Cypel M, Keshavjee S. When to consider lung transplantation for COVID-19. The Lancet Respiratory Medicine. 2020; 8(10): 944-946, doi: 10.1016/s2213-2600(20)30393-3.

9. Chen JY, Qiao K, Liu F, et al. Lung transplantation as therapeutic option in acute respiratory distress syndrome for coronavirus disease 2019-related pulmonary fibrosis. Chin Med J (Engl). 2020; 133(12): 1390-1396, doi: 10.1097/ CM9.0000000000000839, indexed in Pubmed: $\underline{32251003}$.

10. Han W, Zhu M, Chen J, et al. Lung transplantation for elderly patients with end-stage COVID-19 pneumonia. Ann Surg. 2020; 272(1): e33-e34, doi: 10.1097/SLA.0000000000003955, indexed in Pubmed: 32301803.

11. Bharat A, Querrey M, Markov NS. Lung transplantation for patients with severe COVID-19. Science Translational Medicine. 2020; 12(574): eabe4282, doi: 10.1126/scitranslmed.abe4282.

12. Lang C, Jaksch P, Hoda M, et al. Lung transplantation for COVID-19-associated acute respiratory distress syndrome in a PCR-positive patient. The Lancet Respiratory Medicine. 2020; 8(10): 1057-1060, doi: 10.1016/s2213-2600(20)30361-1. 